\title{
A unified Lyapunov function for finite time stabilization of continuous and discontinuous variable structure systems with resets
}

\author{
Harshal B. Oza ${ }^{1}$, Yury V. Orlov ${ }^{2}$ and Sarah K. Spurgeon ${ }^{3}$
}

\begin{abstract}
A unilaterally constrained perturbed double double integrator system is studied in this paper. The aim is to establish uniform finite time stability of the non-linear dynamics in the presence of impacts due to the constraints on the position variable. A non-smooth transformation is utilized to first transform the system into a variable structure system that can be studied within the framework of a conventional discontinuous paradigm. Then, a finite time stable continuous controller is used and stability of the closed-loop dynamics is proven by identifying a new set of Lyapunov functions. The results enable continuous and discontinuous cases to be unified using one parameter that defines the set of Lyapunov functions for each case.
\end{abstract}

\section{INTRODUCTION}

Uniform finite time stabilization of a perturbed double integrator system is considered in the presence of a unilateral constraint on the position variable. Unilaterally constrained systems give rise to hard non-linearities due to jumps or resets in the velocity and are quite challenging from the point of view of stabilization. Such systems occur in various feedback control disciplines [1], [2]. The practical motivation for proposing a synthesis framework for such systems has been demonstrated by applications to biped robots [3], [4], where the generalized velocities of the robot inherently undergo a reset when the swing leg collides with the ground. Studying systems with resets also finds natural application in the area of hybrid systems [5]. Developing a clear understanding of Lyapunov based stability and robustness properties of a closed-loop system when resets with (or without) a finite accumulation point is a challenging and interesting area of study (see reference [6] and references therein for work on hybrid systems and [7], [8] for the area of biped robots).

This paper extends previous work on discontinuous stabilization published in [9] to the case of continuous homogeneous controllers. A recent survey in [10] details the chronology of developments in this are of control engineering. The main feature of the presented work is that it unifies the Lyapunov stability proofs for both the continuous and discontinuous cases while making use of a continuous homogeneous controller. Similar to the work in [11], it is assumed in this paper that the restitution or reset map relating to the velocities just before and after the time of impact is

\footnotetext{
*This work was funded by EPSRC via research grant EP/G053979/1.

${ }^{1}$ Harshal Oza is with Institute of Engineering and Technology, Ahmedabad University, India harshal.ozalahduni.edu. in

${ }^{2}$ Yury V. Orlov is with CICESE, Ensenada, Mexico yorlovecicese.mx

${ }^{3}$ Sarah K. Spurgeon is with School of Engineering and Digital Arts, University of Kent, Canterbury, Kent CT2 7NT, UK S.K.Spurgeon@kent.ac.uk
}

fully known. However, no such assumption is made on the time of impact.

The work presented utilises a continuous homogeneous controller for finite time stabilization of the closed-loop system in the presence of resets. The Zhuravlev-Ivanov non-smooth transformation [12], [13] is utilized to first transform the system into a variable-structure system without jumps. Within engineering applications, this transformation is very useful in the analysis of vibro-impact systems [12], [14]. The resulting transformed system turns out to be a time varying variable structure homogeneous system with a negative homogeneity degree [15] whose solutions are well-defined in the sense of Filippov's definition [16], an attribute absent in the case of the original jump system (see [17] for the solution concept of systems with jumps and friction). It is important to note that the use of finite time stability of switched systems [15] is the most natural method for proving uniform finite time stability due to the variable structure nature of the transformed system despite the continuous controller. This is because all the existing frameworks on continuous homogeneous systems [18], [19], [20], [21] require continuity of the vector field, a condition unavoidably violated at the time of jumps.

The remainder of the paper is organized as follows. Section II provides the mathematical problem formulation including definition of the controller and the non-smooth transformation. Section III motivates the development further by showing mathematically why existing approaches are not suitable when the whole state-space is considered. Section IV identifies a parameterised set of Lyaunov functions to prove step-by-step uniform finite time stability. Section V concludes the paper.

\section{PROBLEM STATEMENT}

Consider the following open loop system:

$$
\begin{aligned}
\dot{x}_{1} & =x_{2} \\
\dot{x}_{2} & =u\left(x_{1}, x_{2}\right)+\omega\left(x_{1}, x_{2}, t\right) \\
x_{1} & \geq 0 \\
x_{2}\left(t_{k}^{+}\right) & =-\bar{e} x_{2}\left(t_{k}^{-}\right) \quad \text { if } \quad x_{2}\left(t_{k}^{-}\right)<0, x_{1}\left(t_{k}\right)=0
\end{aligned}
$$

where $x_{1}, x_{2}$ are the position and the velocity respectively, $u$ is the control input, $\omega\left(x_{1}, x_{2}, t\right)$ is a piece-wise continuous disturbance [15, Sec. 2], [16], $t_{k}$ is the time instant of the $k^{\text {th }}$ jump where the velocity undergoes a reset or jump, $\bar{e}$ represents the loss of energy and $x_{2}\left(t_{k}^{+}\right)$and $x_{2}\left(t_{k}^{-}\right)$represent right and left limits respectively of $x_{2}$ at the jump time $t_{k}$. The equalities (1) and (2) represent the continuous dynamics 
without jumps in the velocity. The inequality (3) represents the unilateral constraint on the position $x_{1}$ which evolves in a domain with a boundary [1]. It is assumed that the jump event occurs instantaneously within an infinitesimally small time and hence mathematically can be represented by Newton's restitution rule [1], [22] given by (4) where it is assumed that $\bar{e} \in(0,1)$.

The piece-wise continuous disturbance $\omega\left(x_{1}, x_{2}, t\right)$ is assumed to admit a uniform upper bound

$$
e s s \sup _{t \geq 0}|\omega(x, t)| \leq M\left|x_{2}\right|^{\alpha}
$$

on its magnitude such that

$$
0<M<\mu_{1}<\mu_{2}-M
$$

The finite time controller [23], [21]

$$
u\left(x_{1}, x_{2}\right)=-\mu_{2}\left|x_{1}\right|^{\frac{\alpha}{2-\alpha}} \operatorname{sign}\left(x_{1}\right)-\mu_{1}\left|x_{2}\right|^{\alpha} \operatorname{sign}\left(x_{2}\right)
$$

is used with $\alpha \in[0,1)$ and $\mu_{2}>\mu_{1}>0$. The aim of the paper is to establish uniform finite-time stability of the closed-loop system (1), (7).

This paper employs Zhuravlev-Ivanov's method of nonsmooth transformation [12], [13], [1, Sec. 1.4.2] to transform the impact system (1) into a jump-free system. Although, this method has been explained for a similar problem in [11], it is included here for completeness. Let the non-smooth coordinate transformation be defined as follows:

$$
\begin{aligned}
& x_{1}=|s|, \quad x_{2}=R v \operatorname{sign}(s), \\
& R=1-k \operatorname{sign}(s v), \quad k=\frac{1-\bar{e}}{1+\bar{e}} .
\end{aligned}
$$

The variable structure transformed system

$$
\begin{aligned}
\dot{s}= & R v \\
\dot{v}= & R^{-1} \operatorname{sign}(s) u(|s|, R v \operatorname{sign}(s)) \\
& +R^{-1} \operatorname{sign}(s) \omega(|s|, R v \operatorname{sign}(s), t)
\end{aligned}
$$

is then obtained by employing (8) and using the dynamics (1), (2). The controller (7) can be represented in the transformed coordinates as follows:

$$
\begin{aligned}
u(|s|, R v \operatorname{sign}(s))= & -\mu_{1}|R v \operatorname{sign}(s)|^{\alpha} \operatorname{sign}(R v \operatorname{sign}(s)) \\
& -\mu_{2}|| s||^{\frac{\alpha}{2-\alpha}} \operatorname{sign}(|s|)
\end{aligned}
$$

Substituting (10) into (9), the closed-loop system in the coordinate frame $(s, v)$ can be obtained as follows:

$$
\begin{aligned}
\dot{s}= & R v \\
\dot{v}= & -\mu_{1} R^{\alpha-1}|v|^{\alpha} \operatorname{sign}(v)-\mu_{2} R^{-1}|s|^{\frac{\alpha}{2-\alpha}} \operatorname{sign}(s) \\
& +R^{-1} \operatorname{sign}(s) \omega(|s|, R v \operatorname{sign}(s), t)
\end{aligned}
$$

Furthermore, the upper-bound (5) can be revised in the new coordinates as follows:

$$
e s s \sup _{t \geq 0}|\omega(s, v, t)| \leq M|R v \operatorname{sign}(s)|^{\alpha}=M R^{\alpha}|v|^{\alpha}
$$

Unlike the existing literature on hybrid systems [5], this paper does not regularize the Zeno motion temporally or dynamically. In the context of the bouncing ball analogy, the temporal regularization as described in [5, Section 4] and subsequently utilized in [24, Section V.C] means that the impact takes a small, but not infinitesimally small, time $\epsilon>0$ and the dynamic regularization means that the impact is elastic but is more like that with a highly stiff spring. No such regularization is employed in this paper and ideal Zeno modes due to non-elastic impacts are allowed giving rise to instantaneous jumps in zero time ${ }^{1}$ Furthermore, existing Lyapunov approaches on the study of 'uniformly small ordinary time' [25] that lead to finite time stabilization results and computation of finite settling time inherently differ in that the jumps in the corresponding Lyapunov function [25, Th. 3.3, Example 3.4] always need to be analyzed at the reset time instant. More importantly, the decrease in successive jumps also have to belong to class $\mathcal{K}_{\infty}$ (see [25, Th. 3.3]). This paper does not need such assumptions while proving robust finite time stability without analyzing the jumps in the Lyapunov function due to the jump-free system produced by the non-smooth transformation.

\section{CONSIDERATION OF EXISTING LYAPUNOV FUNCTIONS}

As noted in [21], the proposed controller (7) is simpler than the controller proposed in [18]. Recall that the control law in the original coordinates is given in [18] as follows:

$$
\begin{aligned}
u\left(x_{1}, x_{2}\right)= & -\operatorname{sign}\left(x_{2}\right)\left|x_{2}\right|^{\alpha} \\
& -\operatorname{sign}\left(\phi_{\alpha}\left(x_{1}, x_{2}\right)\right)\left|\phi_{\alpha}\left(x_{1}, x_{2}\right)\right|^{\frac{\alpha}{2-\alpha}}
\end{aligned}
$$

where, $\phi_{\alpha}\left(x_{1}, x_{2}\right)=x_{1}+\frac{1}{2-\alpha} \operatorname{sign}\left(x_{2}\right)\left|x_{2}\right|^{2-\alpha}$. The closedloop system is then given by (1), (13). The following transformed closed-loop system can be obtained by applying the non-smooth coordinate transformation (8):

$$
\begin{aligned}
\dot{s}= & R v \\
\dot{v}= & R^{-1} \operatorname{sign}(s)(u(|s|, R v \operatorname{sign}(s))) \\
= & -R^{\alpha-1} \operatorname{sign}(v)|v|^{\alpha} \\
& -R^{-1} \operatorname{sign}(s) \operatorname{sign}\left(\phi_{\alpha}\right)\left|\phi_{\alpha}\right|^{\frac{\alpha}{2-\alpha}},
\end{aligned}
$$

where $\phi_{\alpha}(s, v)=|s|+\frac{R^{2-\alpha}}{2-\alpha} \operatorname{sign}(s v)|v|^{2-\alpha}$. Let the Lyapunov function proposed in [18] be defined in the transformed coordinates as follows:

$$
V(s, v)=\frac{2-\alpha}{3-\alpha}\left|\phi_{\alpha}\right|^{\frac{3-\alpha}{2-\alpha}}+r_{2} v \phi_{\alpha}+\frac{r_{1}}{3-\alpha}|v|^{3-\alpha}
$$

where, $r_{1}>1, r_{2}<1$ are arbitrary scalars. The temporal derivative of (15) along the trajectories of the transformed closed-loop system (14) can be obtained as follows:

$$
\begin{aligned}
\dot{V}(s, v)= & -R^{\alpha-1} r_{1} v^{2}-R^{1-\alpha}|v|^{1-\alpha}\left|\phi_{\alpha}\right|^{\frac{1+\alpha}{2-\alpha}} \\
& -R^{-1} r_{2}\left|\phi_{\alpha}\right|^{\frac{2}{2-\alpha}} \operatorname{sign}(s) \\
& -R^{\alpha-1} r_{2} \phi_{\alpha} \operatorname{sign}(v)|v|^{\alpha} \\
& -\left(R^{1-\alpha} r_{2}+R^{-1} r_{1} \operatorname{sign}(s)\right) \operatorname{sign}\left(v \phi_{\alpha}\right)|v|^{2-\alpha}\left|\phi_{\alpha}\right|^{\frac{\alpha}{2-\alpha}}
\end{aligned}
$$

${ }^{1}$ The measure of time is in fact better represented by the Dirac measure [1] and the Dirac distribution. 
Although the homogeneity properties

$$
\begin{aligned}
& V\left(k^{2-\alpha} s, k v\right)=k^{3-\alpha} V(s, v) \\
& \dot{V}\left(k^{2-\alpha} s, k v\right)=k^{2} \dot{V}(s, v)
\end{aligned}
$$

hold true for the transformed system, it is not mathematically correct to restrict the analysis on the closed curve

$$
(s, v): \max _{s, v \neq(0,0)}\left(\left|\phi_{\alpha}\right|^{\frac{1}{2-\alpha}},|v|\right)=1
$$

encircling the origin of the closed-loop system (14) as was done in [18]. This is because it is always possible to have initial conditions either starting from or intersecting with the semi-axis $(s, v): v=0, s<0$ of the transformed system (14) thereby causing (16) to take positive values due to an additional 'sign $(s)$ ' in the third term on the right hand side of (16). This is clearly in contrast to the negative definiteness of the derivative of the Lyapunov function obtained in [18] which enabled analysis to be performed only on the closed curve encircling the origin when combined with the homogeneity property (17).

Thus, motivated by the fact that the Lyapunov framework presented in [18] is applicable neither to the original jumpsystem (1), (13) due to the violation of the continuity requirements at the time of a jump nor to the transformed system (14) due to the non-negative definiteness of the derivative of the Lyapunov function, a proof of finite time stability of the closed-loop system (1), (7) is now presented.

\section{UNIFORM FINITE TIME STABILITY}

The following Lemma is first presented from [9, Lemma 1]:

Lemma 1: Assume $\bar{e} \in(0,1)$, then the following is true:

$$
\operatorname{sign}(s v) \operatorname{sign}\left(R-R^{-1}\right)=-1
$$

It is of interest to note that the discontinuity and in turn Filippov's inclusion [16] in (11) is caused by the fact that $R$ switches between two positive values on sets $\{(s, v): s=$ $0\},\{(s, v): v=0\}$ of Lebesgue measure zero. Let the two values of $R$ be defined as follows:

$$
R= \begin{cases}R_{1}=\frac{2}{1+\bar{e}}, & \text { if } \operatorname{sign}(s v)=-1 \\ R_{2}=\frac{2 \bar{e}}{1+\bar{e}}, & \text { if } \operatorname{sign}(s v)=1 .\end{cases}
$$

Then, it is trivial to note that given $\bar{e} \in(0,1)$, the following is true from the computations in Lemma 1:

$$
\begin{aligned}
& R_{1}>R_{2}>0, R_{1}^{-1}<R_{2}^{-1},\left|R_{1}-R_{1}^{-1}\right|<\left|R_{2}-R_{2}^{-1}\right| \\
& \left|R_{1}-R_{1}^{-1}\right|=\frac{3+\bar{e}}{2}|k|,\left|R_{2}-R_{2}^{-1}\right|=\frac{3 \bar{e}+1}{2 \bar{e}}|k|
\end{aligned}
$$

It is now possible to state the main results of the paper.

Theorem 1: Given $M=0, \alpha \in(0,1)$, the impact system (1), (2), (3), (4), (7) and its transformed version (11) are globally finite time stable.

Proof: Lyapunov stability analysis can be performed in the transformed coordinates since both represent the same system. Let a Lyapunov function candidate be given as follows:

$$
V(s, v)=\mu_{2} \frac{2-\alpha}{2}|s|^{\frac{2}{2-\alpha}}+\frac{1}{2} v^{2}
$$

Note that the function $V(s, v)$ is a globally radially unbounded $\mathcal{C}^{1}$ smooth positive definite function. By computing the temporal derivative of this function along the system trajectories of (11) with $M=0$, it is obtained that,

$$
\begin{aligned}
\dot{V} & \leq \mu_{2}|v||s|^{\frac{\alpha}{2-\alpha}}\left|R-R^{-1}\right| \operatorname{sign}(s v) \operatorname{sign}\left(R-R^{-1}\right) \\
& -\mu_{1} R^{\alpha-1}|v|^{\alpha+1}
\end{aligned}
$$

From Lemma 1, (23) can be simplified as follows:

$$
\dot{V} \leq-\mu_{2}|v||s|^{\frac{\alpha}{2-\alpha}}\left|R-R^{-1}\right|-\mu_{1} R^{\alpha-1}|v|^{\alpha+1}
$$

It can be verified that $R^{\alpha-1}>0$ for either sign of $\operatorname{sign}(s v)$ since $\bar{e} \in(0,1)$. Furthermore, the trajectory of the closed-loop system (11) in the $(s, v)$ plane never generates a sliding mode on $v=0$ since $v \dot{v} \nless 0$. Since, the equilibrium point $s=v=0$ is the only trajectory of (11) on the invariance manifold $v=0$ where $\dot{V}(s, v)=0$ and since (24) holds true for almost all $t$, the differential inclusion (11) is globally equiuniformly asymptotically stable by applying the invariance principle [26], [27]. Moreover, the system described in (11) is globally homogeneous of negative degree $q=-1$ with respect to the dilation $r=\left(\frac{2-\alpha}{1-\alpha}, \frac{1}{1-\alpha}\right)$ and is globally equiuniformly finite time stable according to $[15$, Theorem 3.1].

The closed-loop system (11) is a globally homogeneous system if $\omega(x, t)=0 \forall t \geq 0$. Consider next the case when $M$ takes a nonzero value. The control law (10) can reject any disturbance $\omega$ with a uniform upper bound (12). Global asymptotic stability was established in [21]. However, to establish finite time stability, uniform asymptotic stability is required [15]. The next theorem achieves this objective for the transformed system (11) (and equivalently for the jump system (1)-(4), (7)).

Theorem 2: Given $\alpha \in\left(\frac{1}{2}, 1\right)$, the closed-loop impact system (1)-(4), (7) and its transformed version (11) are globally equiuniformly finite time stable, regardless of whichever disturbance $\omega$, satisfying condition (12) (or equivalently (5)) with $M<\mu_{1}<\mu_{2}$, affects the system.

Proof: the proof is given in several steps.

1. Global Asymptotic Stability Under the conditions of the theorem, the time derivative of the Lyapunov function (22), computed along the trajectories of (11) is estimated as follows:

$$
\dot{V} \leq-\mu_{2}|v||s|^{\frac{\alpha}{2-\alpha}}\left|R-R^{-1}\right|-\left(\mu_{1}-M\right) R^{\alpha-1}|v|^{\alpha+1}
$$

The first term on the right hand side of (25) follows from Lemma 1 . Since $M<\mu_{1}$ by a condition of the Theorem, the global asymptotic stability of (11) is then established by applying the invariance principle [26], [27] as discussed in Theorem 1.

\section{Semiglobal Strong Lyapunov Functions.}

The goal of this step is to show the existence of a parameterized family of local Lyapunov functions $V_{\tilde{R}}(s, v)$, with an a-priori given $\tilde{R}>0$, such that each $V_{\tilde{R}}(s, v)$ is well-posed on the corresponding compact set

$$
D_{\tilde{R}}=\left\{(s, v) \in \mathbb{R}^{2}: V(s, v) \leq \tilde{R}\right\} .
$$


In other words, $V_{\tilde{R}}(s, v)$ is to be positive definite on $D_{\tilde{R}}$ and its derivative, computed along the trajectories of the uncertain system (11) with initial conditions within $D_{\tilde{R}}$, is to be negative definite in the sense that,

$$
\dot{V}_{\tilde{R}}(s, v) \leq-W_{\tilde{R}}(s, v)
$$

for all $(s, v) \in D_{\tilde{R}}$ and for some $W_{\tilde{R}}(s, v)$, positive definite on $D_{\tilde{R}}$. A parameterized family of Lyapunov functions $V_{\tilde{R}}(s, v), \tilde{R}>0$, with the properties defined above are constructed by combining the Lyapunov function $V$ of (22), whose time derivative along the system motion is only negative semi-definite, with the indefinite function

$$
\begin{aligned}
U(s, v) & =U_{1}(s, v)+U_{2}(s, v) \\
& =s v|v|^{\alpha}+\kappa_{1} \alpha s^{3} v,
\end{aligned}
$$

as follows:

$$
\begin{aligned}
V_{\tilde{R}}(s, v)= & V(s, v)+\kappa_{\tilde{R}} U(s, v) \\
= & \mu_{2} \frac{2-\alpha}{2}|s|^{\frac{2}{2-\alpha}}+\frac{1}{2} v^{2}+ \\
& \kappa_{\tilde{R}}\left(s v|v|^{\alpha}+\kappa_{1} \alpha s^{3} v\right),
\end{aligned}
$$

where the weight parameters $\kappa_{\tilde{R}}, \kappa_{1}$ are chosen small enough, namely,

$$
\begin{aligned}
& \kappa_{\tilde{R}}<\min \left\{\begin{array}{cl}
\frac{1}{(\sqrt{2 \tilde{R}})^{2 \alpha}+\kappa_{1} \alpha}, & \frac{(2-\alpha) \mu_{2}}{\rho^{(1-\alpha)}\left(1+\kappa_{1} \alpha \rho^{2(2-\alpha)}\right)}, \\
\frac{\mu_{2}\left|R_{1}-R_{1}^{-1}\right|}{\Theta}, & \frac{\mu_{1}-M}{R_{1} \sqrt{2 \tilde{R}}}
\end{array}\right\} \\
& \kappa_{1}<\frac{\mu_{2} R_{1}^{-1}(1+\alpha)}{3 \alpha\left(\mu_{1}+M\right) R_{1}^{\alpha-1} \rho^{\frac{4-3 \alpha}{2}} \sqrt{2 \tilde{R}}}
\end{aligned}
$$

where,

$$
\begin{aligned}
\rho= & \frac{2 \tilde{R}}{(2-\alpha) \mu_{2}} \\
\Theta= & \left(\mu_{1}+M\right)(1+\alpha) R_{1}^{\alpha-1}(2 \tilde{R})^{\frac{2 \alpha-1}{2}} \rho^{1-\alpha}+ \\
& 3 \kappa_{1} \alpha R_{1} \rho^{\frac{4-3 \alpha}{2}} \sqrt{2 \tilde{R}}
\end{aligned}
$$

and $R_{1}$ is defined in (20). Noting that, due to (25), all possible solutions of the uncertain system (11), initialized at $t_{0} \in \mathbb{R}$ within the compact set (26), are a-priori estimated by

$$
\sup _{t \in\left[t_{0}, \infty\right)} V(s, v) \leq \tilde{R},
$$

the following inequalities hold true:

$$
|s|^{\frac{2}{2-\alpha}} \leq \rho, \quad|v| \leq \sqrt{2 \tilde{R}} .
$$

The Lyapunov function (29) is positive definite on the compact set (26), for all $(s, v) \in D_{\tilde{R}} \backslash\{0,0\}$ and $\kappa_{\tilde{R}}>0$ satisfying (30) as shown below:

$$
\begin{aligned}
V_{\tilde{R}}(s, v)= & \mu_{2} \frac{2-\alpha}{2}|s|^{\frac{2}{2-\alpha}}+\frac{1}{2} v^{2}+\kappa_{\tilde{R}} s v|v|^{\alpha}+\kappa_{\tilde{R}} \kappa_{1} \alpha s^{3} v \\
\geq & \mu_{2} \frac{2-\alpha}{2}|s|^{\frac{2}{2-\alpha}}+\frac{1}{2} v^{2}-\frac{1}{2} \kappa_{\tilde{R}} s^{2}-\frac{1}{2} \kappa_{\tilde{R}}|v|^{2 \alpha} v^{2} \\
& -\frac{1}{2} \kappa_{\tilde{R}} \kappa_{1} \alpha s^{6}-\frac{1}{2} \kappa_{\tilde{R}} \kappa_{1} \alpha v^{2} \\
\geq & L_{\tilde{R}} V(s, v)
\end{aligned}
$$

where,

$L_{\tilde{R}}<\min \left\{\begin{array}{c}\mu_{2} \frac{2-\alpha}{2}-\frac{1}{2} \kappa_{\tilde{R}} \rho^{(1-\alpha)}\left(1+\kappa_{1} \alpha \rho^{2(2-\alpha)}\right), \\ 1-\kappa_{\tilde{R}}\left(\sqrt{2 \tilde{R}}^{2 \alpha}+\kappa_{1} \alpha\right)\end{array}\right\}$,

the trivial inequality $2 a b>-\left(a^{2}+b^{2}\right), \forall a, b \in \mathbb{R}$, the equalities

$$
s^{6}=|s|^{\frac{2(1-\alpha)}{2-\alpha}}|s|^{\frac{2}{2-\alpha}}|s|^{4}, \quad|v|^{2(\alpha+1)}=v^{2}|v|^{2 \alpha}
$$

and the bound (33) are utilized. It should be noted that $L_{\tilde{R}}>$ 0 due to (30) and hence positive definiteness of $V_{\tilde{R}}$ is ensured from (34) on $D_{\tilde{R}} \backslash\{0,0\}$. Similarly it can be shown that the following inequality holds:

$$
V_{\tilde{R}}(s, v) \leq M_{\tilde{R}} V(s, v)
$$

where,

$M_{\tilde{R}}>\max \left\{\begin{array}{l}\mu_{2} \frac{2-\alpha}{2}+\frac{1}{2} \kappa_{\tilde{R}} \rho^{(1-\alpha)}\left(1+\kappa_{1} \alpha \rho^{2(2-\alpha)}\right), \\ 1+\kappa_{\tilde{R}}\left(\sqrt{2 \tilde{R}}+\kappa_{1} \alpha\right)\end{array}\right\}$

The time derivative of the indefinite function $U(s, v)$ along the trajectories of the uncertain system (11) is obtained as follows:

$$
\begin{aligned}
\dot{U}_{1}(s, v)= & R|v|^{\alpha+2}+s|v|^{\alpha}\left(-\mu_{1} R^{\alpha-1}|v|^{\alpha} \operatorname{sign}(v)\right) \\
+ & s|v|^{\alpha}\left(-\mu_{2} R^{-1}|s|^{\frac{\alpha}{2-\alpha}} \operatorname{sign}(s)\right) \\
+ & s|v|^{\alpha} R^{-1} \operatorname{sign}(s) \omega(s, v, t) \\
& +\alpha s v|v|^{\alpha-1} \operatorname{sign}(v)\left(-\mu_{1} R^{\alpha-1}|v|^{\alpha} \operatorname{sign}(v)\right) \\
& +\alpha s v|v|^{\alpha-1} \operatorname{sign}(v)\left(-\mu_{2} R^{-1}|s|^{\frac{\alpha}{2-\alpha}} \operatorname{sign}(s)\right) \\
& +\alpha s v|v|^{\alpha-1} \operatorname{sign}(v) R^{-1} \operatorname{sign}(s) \omega(s, v, t) \\
\leq & R|v|^{\alpha+2}+\left(\mu_{1}+M\right)(1+\alpha) R^{\alpha-1}|s||v|^{2 \alpha} \\
& -\mu_{2}(1+\alpha) R^{-1}|s|^{\frac{2}{2-\alpha}}|v|^{\alpha}
\end{aligned}
$$

Similarly,

$$
\begin{aligned}
\dot{U}_{2}(s, v) & =3 \kappa_{1} \alpha R s^{2} v^{2}+\kappa_{1} \alpha s^{3}\left(-\mu_{1} R^{\alpha-1}|v|^{\alpha} \operatorname{sign}(v)\right) \\
& +\kappa_{1} \alpha s^{3}\left(-\mu_{2} R^{-1}|s|^{\frac{\alpha}{2-\alpha}} \operatorname{sign}(s)\right) \\
& +\kappa_{1} \alpha s^{3} R^{-1} \operatorname{sign}(s) \omega(s, v, t) \\
\leq & 3 \kappa_{1} \alpha R s^{2} v^{2}+\kappa_{1} \alpha\left(\mu_{1}+M\right) R^{\alpha-1}|s|^{3}|v|^{\alpha} \\
& -\kappa_{1} \alpha R^{-1} \mu_{2}|s|^{\frac{6-2 \alpha}{2-\alpha}}
\end{aligned}
$$

It should be noted that the inequality

$$
\begin{aligned}
& |s|=|s|^{\frac{2(1-\alpha)}{2-\alpha}}|s|^{\frac{\alpha}{2-\alpha}} \leq \rho^{1-\alpha}|s|^{\frac{\alpha}{2-\alpha}}, \\
& |v|^{2 \alpha}=|v||v|^{2 \alpha-1} \leq|v| \sqrt{2 \tilde{R}}^{2 \alpha-1}
\end{aligned}
$$

holds true due to the condition $\alpha \in\left(\frac{1}{2}, 1\right)$ of the Theorem. The last inequalities of (37) and (38) are re-written by utilizing (33) and (39) as follows:

$$
\begin{aligned}
\dot{U}_{1}(s, v) \leq & \left(\mu_{1}+M\right)(1+\alpha) R^{\alpha-1}|s|^{\frac{\alpha}{2-\alpha}}|v| \sqrt{2 \tilde{R}}^{2 \alpha-1} \rho^{1-\alpha} \\
& +R|v|^{\alpha+1} \sqrt{2 \tilde{R}}-\mu_{2}(1+\alpha) R^{-1}|s|^{\frac{2}{2-\alpha}}|v|^{\alpha}(40) \\
\dot{U}_{2}(s, v) \leq & 3 \kappa_{1} \alpha R|s|^{\frac{\alpha}{2-\alpha}}|v| \rho^{\frac{4-3 \alpha}{2}} \sqrt{2 \tilde{R}} \\
& +\kappa_{1} \alpha\left(\mu_{1}+M\right) R^{\alpha-1}|s|^{3}|v|^{\alpha} \\
& -\kappa_{1} \alpha \mu_{2} R^{-1}|s|^{\frac{6-2 \alpha}{2-\alpha}}
\end{aligned}
$$

where the corresponding upper bound on $|v|$ and $|s|$ from (33) are utilized. The parameter $R$ in (25) and (40) is a 
state function and keeps switching between the two values as shown in Lemma 1. This corresponds to the fact that the rate of decay of the Lyapunov function (29) switches depending on $R$. Hence, by combining (25) and (40) and by considering the slowest decay by utilizing (21), the time derivative of (29) can be obtained as follows:

$$
\begin{aligned}
\dot{V}_{\tilde{R}} \leq & -\beta_{1}|v||s|^{\frac{\alpha}{2-\alpha}}-\beta_{2}|v|^{\alpha+1}-\kappa_{\tilde{R}} \beta_{3}|v|^{\alpha}|s|^{\frac{2}{2-\alpha}} \\
& -\kappa_{\tilde{R}} \kappa_{1} \alpha \mu_{2} R^{-1}|s|^{\frac{6-2 \alpha}{2-\alpha}}
\end{aligned}
$$

where

$$
\begin{aligned}
& \beta_{1}=\mu_{2}\left|R_{1}-R_{1}^{-1}\right|-\kappa_{\tilde{R}} \Theta \\
& \beta_{2}=\left(\mu_{1}-M\right) R_{1}^{\alpha-1}-\kappa_{\tilde{R}} R_{1} \sqrt{2 \tilde{R}} \\
& \beta_{3}=\mu_{2} R_{1}^{-1}(1+\alpha)-\kappa_{1} \alpha\left(\mu_{1}+M\right) R_{1}^{\alpha-1} \rho^{\frac{4-3 \alpha}{2}} \sqrt{2 \tilde{R}},
\end{aligned}
$$

(33) and the equality $|s|^{3}=|s|^{\frac{2}{2-\alpha}}|s|^{\frac{4-3 \alpha}{2-\alpha}}$ are utilised. It should be noted that the expressions $\beta_{1}>0, \beta_{2}>0, \beta_{3}>0$ hold true due to (30).

Ignoring the negative semi-definite terms with $\beta_{1}, \beta_{3}$, (41) can be rewritten as follows:

$$
\dot{V}_{\tilde{R}} \leq-\beta_{2}|v|^{\alpha+1}-\kappa_{\tilde{R}} \kappa_{1} \alpha \mu_{2} R^{-1}|s|^{\frac{6-2 \alpha}{2-\alpha}}
$$

Furthermore, the following inequalities hold true within the compact set (26):

$$
\begin{gathered}
v^{2}=|v|^{2}=|v|^{\alpha+1}|v|^{1-\alpha} \leq|v|^{\alpha+1}(\sqrt{2 \tilde{R}})^{1-\alpha} \\
\Rightarrow-|v|^{\alpha+1} \leq-\frac{v^{2}}{(\sqrt{2 \tilde{R}})^{1-\alpha}}
\end{gathered}
$$

Hence, (43) can be simplified as follows:

$$
\dot{V}_{\tilde{R}} \leq-c_{\tilde{R}}\left[|s|^{\frac{6-2 \alpha}{2-\alpha}}+v^{2}\right]
$$

where,

$$
c_{\tilde{R}}=\min \left\{\frac{\beta_{2}}{(\sqrt{2 \tilde{R}})^{1-\alpha}}, \quad \kappa_{\tilde{R}} \kappa_{1} \alpha \mu_{2} R_{1}^{-1}\right\}>0
$$

Case 1: $|s| \geq 1$ :

The following inequality holds true for $|s| \geq 1$ :

$$
\frac{6-2 \alpha}{2-\alpha} \geq \frac{2}{2-\alpha} \Leftrightarrow|s|^{\frac{6-2 \alpha}{2-\alpha}} \geq|s|^{\frac{2}{2-\alpha}}
$$

Also, the following can be obtained from (36):

$$
\frac{M_{\tilde{R}}}{2} \max \left\{1, \mu_{2}(2-\alpha)\right\}\left(|s|^{\frac{2}{2-\alpha}}+v^{2}\right) \geq V_{\tilde{R}}(s, v)
$$

Hence, the following inequality is then obtained for $|s| \geq 1$ by combining (45), (47) and (48):

$$
\dot{V}_{\tilde{R}} \leq-\bar{\kappa}_{1} V_{\tilde{R}}
$$

where,

$$
\bar{\kappa}_{1}=\frac{2 c_{\tilde{R}}}{M_{\tilde{R}} \max \left\{1, \mu_{2}(2-\alpha)\right\}} .
$$

Case 2: $|s|<1$ :
Noting that the following inequalities hold true for $|s|<1$ :

$$
|s|^{\frac{6-2 \alpha}{2-\alpha}}>|s|^{\frac{2 \gamma}{2-\alpha}} \Leftrightarrow \frac{6-2 \alpha}{2-\alpha}<\frac{2 \gamma}{2-\alpha} \Leftrightarrow \gamma>3-\alpha,
$$

for some $\gamma>3-\alpha$. As $3-\alpha<\frac{5}{2}$ always holds true due to $\alpha \in\left(\frac{1}{2}, 1\right), \gamma \geq \frac{5}{2}$ is a valid choice. In the following, $\gamma=3$ is chosen. It can be seen that the following equality holds:

$$
\begin{aligned}
\left(|s|^{\frac{2}{2-\alpha}}+v^{2}\right)^{3} & =|s|^{\frac{6}{2-\alpha}}+3|s|^{\frac{4}{2-\alpha}} v^{2}+3|s|^{\frac{2}{2-\alpha}} v^{4}+v^{6} \\
& \leq \max \left\{\rho^{\alpha}, K_{1}\right\}\left(|s|^{\frac{6-2 \alpha}{2-\alpha}}+v^{2}\right)
\end{aligned}
$$

where the bounds (33) has been utilised resulting in the following definition of $K_{1}$ :

$$
K_{1}=\max \left\{3 \rho^{2}, 3 \rho(2 \tilde{R}),(2 \tilde{R})^{2}\right\}>0
$$

Note that the following can be obtained from (36):

$$
\left(\frac{M_{\tilde{R}}}{2} \max \left\{1, \mu_{2}(2-\alpha)\right\}\left(|s|^{\frac{2}{2-\alpha}}+v^{2}\right)\right)^{3} \geq\left(V_{\tilde{R}}(s, v)\right)^{3}
$$

Then, combining (45), (52) and (54):

$$
\dot{V}_{\tilde{R}}(s, v) \leq-c_{\tilde{R}}\left(|s|^{\frac{6-2 \alpha}{2-\alpha}}+v^{2}\right) \leq-\bar{\kappa}\left(V_{\tilde{R}}\right)^{3}
$$

where,

$$
\bar{\kappa}=\frac{c_{\tilde{R}}}{\left(\frac{M_{\tilde{R}}}{2} \max \left\{1, \mu_{2}(2-\alpha)\right\}\right)^{3} \max \left\{\rho^{\alpha}, K_{1}\right\}}>0
$$

Hence, the desired uniform negative definiteness (27) is obtained by combining (49) and (55) as follows:

$$
W_{\tilde{R}}(s, v)=\min \left\{\bar{\kappa}_{1} V_{\tilde{R}}, \bar{\kappa}\left(V_{\tilde{R}}\right)^{3}\right\}
$$

\section{Global Equiuniform Asymptotic Stability}

Since the inequality (55) holds on the solutions of the uncertain system (11), initialized within the compact set (26), the decay of the function $V_{\tilde{R}}(s, v)$ can be found by considering the majorant solution $\nu(t)$ of $V_{\tilde{R}}$ as follows:

$$
\dot{\nu}(t)= \begin{cases}-\bar{\kappa}_{1} \nu, & |s| \geq 1 \\ -\bar{\kappa}_{2} \nu \gamma, & |s|<1\end{cases}
$$

where, $\gamma>3-\alpha$ is introduced for generality. A more conservative decay than that in (58) can be computed. There are two possible sub-cases, namely, $\nu(t) \geq 1$ and $\nu(t)<1$ for each of the cases $|s| \geq 1$ and $|s|<1$. The following expressions hold true for a positive definite function $\nu(t)$ and a scalar $\gamma>1$ :

$$
\begin{array}{ll}
\nu(t)^{\gamma} \geq \nu(t) \Rightarrow-\nu(t)^{\gamma} \leq-\nu(t) & \text { if } \nu(t) \geq 1 \\
\nu(t)^{\gamma} \leq \nu(t) \Rightarrow-\nu(t) \leq-\nu(t)^{\gamma} & \text { if } \nu(t)<1 .
\end{array}
$$

Hence, the decay (58) is modified by utilising (59) independent of the magnitude of $|s|$ and dependent on $\nu(t)$ as follows :

$$
\dot{\nu}(t)= \begin{cases}-\bar{\kappa} \nu, & \text { if } \nu(t) \geq 1 \\ -\bar{\kappa} \nu^{\gamma}, & \text { if } \nu(t)<1\end{cases}
$$


where,

$$
\bar{\kappa}=\min \left\{\bar{\kappa}_{1}, \bar{\kappa}_{2}\right\} \quad>0 .
$$

The solution for the case $\nu(t)<1$ can be integrated as follows:

$$
\int_{\nu_{0}}^{\nu(t)} \frac{\mathrm{d} \nu(t)}{\nu^{\gamma}}=-\bar{\kappa} \int_{t_{0}}^{t} \mathrm{~d} t
$$

where $\nu_{0}=\nu\left(t_{0}\right)$. The general solution of $\nu(t)$ of (60) can then be obtained by utilising (60) and by combining the solutions of both (49) and (62):

$$
\nu(t)= \begin{cases}\nu\left(t_{0}\right) e^{-\bar{\kappa}\left(t-t_{0}\right)}, & \text { if } \nu(t) \geq 1 \\ \nu\left(t_{1}\right)\left(\frac{1}{\bar{\kappa}\left(t-t_{1}\right)(\gamma-1) \nu_{0}^{\gamma-1}+1}\right)^{\frac{1}{\gamma-1}}, & \text { if } \nu(t)<1 .\end{cases}
$$

It can be easily seen that the solution $\nu(t) \rightarrow 0$ as $t \rightarrow \infty$ and that the decay rate depends on the gain parameters $\mu_{1}, \mu_{2}$, bound $M$ on the disturbance $\omega$ and the system property $R_{1}$. On the compact set (26), the following inequality holds (see (34), (36)):

$$
L_{\tilde{R}} V(s, v) \leq V_{\tilde{R}}(s, v) \leq M_{\tilde{R}} V(s, v)
$$

for all $(s, v) \in D_{\tilde{R}}$ and positive constants $L_{\tilde{R}}, M_{\tilde{R}}$. The above inequalities (63) and (64) ensure that the globally radially unbounded function $V\left(x_{1}, x_{2}\right)$ decays exponentially

$$
\begin{aligned}
& V(s(t), v(t)) \leq \\
& \begin{cases}L_{\tilde{R}}^{-1} M_{\tilde{R}} \tilde{R} e^{-\bar{\kappa}\left(t-t_{0}\right)}, & \text { if } V_{\tilde{R}} \geq 1 ; \\
L_{\tilde{R}}^{-1} M_{\tilde{R}} \tilde{R}\left(\frac{1}{\bar{\kappa}\left(t-t_{1}\right)(\gamma-1) \nu_{0}^{\gamma-1}+1}\right)^{\frac{1}{\gamma-1}}, & \text { if } V_{\tilde{R}}<1 .\end{cases}
\end{aligned}
$$

on the solutions of (11) uniformly in $\omega$ and the initial data, located within an arbitrarily large set (26). This proves that the uncertain system (11) is globally equiuniformly asymptotically stable around the origin $(s, v)=(0,0)$.

\section{Global Equiuniform Finite Time Stability.}

Due to (12), the piece-wise continuous uncertainty $R_{1}^{\alpha-1} \omega(t) \operatorname{sign}(s)$ on the right hand side of the system (11) is locally uniformly bounded by $R_{1}^{\alpha-1} M|v|^{\alpha}$ whereas the remaining part of the feedback is globally homogeneous with homogeneity degree $q=-1$ with respect to dilation $r=\left(\frac{2-\alpha}{1-\alpha}, \frac{1}{1-\alpha}\right)$. It remains to verify, however, whether the existing quasi-homogeneity result [15, Theorem 3.2] can be extended to the continuous case in question. Let the piece-wise continuous function $R_{1}^{\alpha-1} \omega(s, v, t) \operatorname{sign}(s)=$ $R_{1}^{\alpha-1} \omega^{c}(s, v, t) \operatorname{sign}(s)$ be defined for an arbitrary $c \geq$ $\max \left\{1, c_{0}\right\}$, where $c_{0}$ is lower homogeneity parameter, as follows:

$$
\omega^{c}(s, v, t)=c^{q+r_{2}} \omega\left(c^{-r_{1}} s, c^{-r_{2}} v, c^{q} t\right)
$$

where, the right hand side represents a parameterised set of uncertainties. Then the following holds true:

$$
\begin{aligned}
\left|\omega^{c}(s, v, t)\right| & =\left|c^{q+r_{2}} \omega\left(c^{-r_{1}} s, c^{-r_{2}} v, c^{q} t\right)\right| \\
\left|\omega^{c}(s, v, t)\right| & \leq c^{q+r_{2}} M R^{\alpha}\left|c^{-r_{2}} v\right|^{\alpha} \\
& \leq c^{q+r_{2}-\alpha r_{2}} M R^{\alpha}|v|^{\alpha}
\end{aligned}
$$

Hence all parameterised uncertainties represented by the right hand side of (66) are admissible in the sense of (12) if the following holds true:

$$
c^{q+r_{2}-\alpha r_{2}} \leq 1
$$

From the definitions $r_{2}=\frac{1}{1-\alpha}, q=-1$, it is obtained that

$$
q+r_{2}-\alpha r_{2}=0 \Rightarrow c^{q+r_{2}-\alpha r_{2}} \leq 1
$$

Hence, recalling from [15, Definitions 2.9, 2.10], the solutions $s^{c}(t)=c^{r_{1}} s\left(c^{q} t\right), v^{c}(t)=c^{r_{2}} v\left(c^{q} t\right)$ are solutions of the system (11) with the piece-wise continuous function $\omega(s, v, t)=\omega^{c}(s, v, t)$. Hence, any solution of the differential equation (11) evolving within a homogeneity ball $B_{\delta}$, generates a parameterised set of solutions $s^{c}(t)$, $v^{c}(t)$ with the parameter $c$ large enough. Hence, (11) is locally homogeneous of degree $q=-1$ with the dilation $\left(r_{1}, r_{2}\right)=\left(\frac{2-\alpha}{1-\alpha}, \frac{1}{1-\alpha}\right)$. Thus, the globally equiuniformly asymptotically stable system (11) and in turn the original impact system (1), (7) are globally equiuniformly finite time stable according to [15, Theorem 3.1].

\section{CONCLUSION}

It can be seen from the results presented that setting $\alpha=0$ is admissible by the main claims of this paper. The resulting analysis produces a discontinuous controller and a non-smooth Lyapunov function given by (7) and (22) respectively. The presented Lyapunov analysis then coincides with recent results in [11] as can be seen from (28) which produces $U(s, v)=s v$ as given in [11]. Hence, this paper is a generalization of variable structure systems parameterised by $\alpha$ both in the sense of encompassing continuous and discontinuous right hand sides as well as in the sense of unifying the Lyapunov analysis when resets are present in the dynamics.

\section{REFERENCES}

[1] B. Brogliato, Nonsmooth Impact Mechanics. London: Springer Verlag, 1996.

[2] R. Goebel, R. Sanfelice, and A. Teel, "Hybrid dynamical systems," Control Systems, IEEE, vol. 29, no. 2, pp. 28 -93, 2009.

[3] C. Chevallereau, G. Abba, Y. Aoustin, F. Plestan, E. Westervelt, C. Canudas-De-Wit, and J. Grizzle, "Rabbit: a testbed for advanced control theory," Control Systems Magazine, IEEE, vol. 23, no. 5, pp. $57-79,2003$.

[4] E. Westervelt, J. Grizzle, C. Chevallereau, J. Choi, and B. Morris, Feedback Control of Dynamic Bipedal Robot Locomotion, ser. Automation and Control Engineering. CRC Press, Taylor and Francis Ltd, June 2007.

[5] K. H. Johansson, M. Egerstedt, J. Lygeros, and S. Sastry, "On the regularization of Zeno hybrid automata," Systems \& Control Letters, vol. 38, no. 3, pp. $141-150,1999$.

[6] M. O'Toole and E. Navarro-Lpez, "A hybrid automaton for a class of multi-contact rigid-body systems with friction and impacts," in 4th IFAC Conference on Analysis and Design of Hybrid Systems, 2012, pp. $298-306$.

[7] J. Grizzle, J. Choi, H. Hammouri, and B. Morris, "On observerbased feedback stabilization of periodic orbits in bipedal locomotion," Methods and Models in Automation and Robotics (MMAR 2007), Szczecin, Poland. Plenary Talk, 2007.

[8] Y. Aoustin, C. Chevallereau, and Y. Orlov, "Finite time stabilization of a perturbed double integrator - part ii: applications to bipedal locomotion," in 49th IEEE Conference on Decision and Control (CDC), 2010, pp. $3554-3559$. 
[9] H. B. Oza, Y. V. Orlov, and S. K. Spurgeon, "Finite time stabilization of a perturbed double integrator with unilateral constraints," Mathematics and Computers in Simulation, vol. 95, pp. 200 - 212, 2014, discontinuous Differential Systems : Theory and Numerical Methods. [Online]. Available: http://www.sciencedirect.com/science/article/pii/S0378475412001164

[10] M. Kawski, "Homogeneity in control: Geometry and applications," in European Control Conference (ECC), 2015, pp. 2449-2457.

[11] H. Oza, Y. Orlov, and S. Spurgeon, "Finite time stabilization of perturbed double integrator with jumps in velocity," in 50th IEEE Conference on Decision and Control and European Control Conference (CDC-ECC), 2011, pp. $4610-4615$.

[12] V. F. Zhuravlev, "Equations of motion of mechanical systems with ideal onesided links : Pmm vol. 42, no. 5, 1978. pp 781-788," Journal of Applied Mathematics and Mechanics, vol. 42, no. 5, pp. 839 - 847, 1978.

[13] A. P. Ivanov, "Analytical methods in the theory of vibro-impact systems," Journal of Applied Mathematics and Mechanics, vol. 57, no. 2, pp. $221-236,1993$.

[14] K. Avramov, "Application of nonsmooth transformations to analyze a vibroimpact duffing system," International Applied Mechanics, vol. 44, pp. 1173-1179, 2008.

[15] Y. Orlov, "Finite time stability and robust control synthesis of uncertain switched systems," SIAM Journal on Control and Optimization, vol. 43, no. 4, pp. 1253-1271, 2005.

[16] A.F.Filippov, Differential Equations with Discontinuous Right hand Sides, ser. Mathematics and its Applications. Springer, 1988, vol. 18.

[17] D. Stewart, "Rigid-body dynamics with friction and impact," SIAM Review, vol. 42, no. 1, pp. 3-39, 2000.

[18] S. Bhat and D. Bernstein, "Continuous finite-time stabilization of the translational and rotational double integrators," IEEE Transactions on Automatic Control, pp. 678 -682, 1998.

[19] V. T. Haimo, "Finite time controllers," SIAM Journal on Control and Optimization, vol. 24, no. 4, pp. 760-770, 1986.

[20] Y. Hong, J. Huang, and Y. Xu, "On an output feedback finitetime stabilization problem," IEEE Transactions on Automatic Control, vol. 46, no. 2, pp. $305-309,2001$.
[21] Y. Orlov, Y. Aoustin, and C. Chevallereau, "Finite time stabilization of a perturbed double integrator - part I: Continuous sliding mode-based output feedback synthesis," IEEE Transactions on Automatic Control, vol. 56 , no. 3,2010 .

[22] J. Grizzle, G. Abba, and F. Plestan, "Asymptotically stable walking for biped robots: analysis via systems with impulse effects," IEEE Transactions on Automatic Control, vol. 46, no. 1, pp. 51 -64, 2001.

[23] S. Bhat and D. Bernstein, "Finite-time stability of homogeneous systems," in Proceedings of the American Control Conference, 1997, pp. $2513-2514$.

[24] C. Cai, R. Goebel, and A. Teel, "Smooth Lyapunov functions for hybrid systems part ii: (pre)asymptotically stable compact sets," IEEE Transactions on Automatic Control, vol. 53, no. 3, pp. 734 -748, 2008.

[25] R. Goebel and A. Teel, "Lyapunov characterization of Zeno behavior in hybrid systems," in 47th IEEE Conference on Decision and Control(CDC), 2008, pp. $2752-2757$.

[26] J. Alvarez, Y. Orlov, and L. Acho, "An invariance principle for discontinuous dynamic systems with applications to a coulumb friction oscillator," Journal of Dynamic Systems, Measurement and Control, vol. 74, pp. 190 - 198, 2000.

[27] D. Shevitz and B. Paden, "Lyapunov stability theory of nonsmooth systems," IEEE Transactions on Automatic Control, vol. 39, no. 9, pp. $1910-1914,1994$. 